\title{
Glucose enhances long-term declarative memory in mildly head-injured varsity rugby players
}

\author{
JACQUELINE A. PETTERSEN and RONALD W. SKELTON \\ University of Victoria, Victoria, British Columbia, Canada
}

\begin{abstract}
In the present investigation, we sought to determine whether glucose could enhance declarative memory in head-injured individuals, as has been shown previously with elderly and Alzheimer's patients who had preexisting memory impairments. Varsity rugby players, both with and without a history of concussions, were given glucose- or saccharin-sweetened beverages and then tested on a series of neuropsychological tests of attention and memory; their blood-glucose levels were monitored. Beverages and tests were administered in a counterbalanced, crossover design, enabling within-subjects comparisons. Previously concussed participants were found to perform slightly worse than participants with no history of concussions (controls) on the preliminary screening interview tests and tests of memory and divided attention given in the saccharin (placebo) condition. Glucose was shown to enhance performance of the previously concussed participants and impair the performance of controls on tests of long-term declarative memory. The effects of glucose on memory appeared to be related to the participants' ability to clear glucose from the blood.
\end{abstract}

Head injuries and resulting cognitive impairments represent a significant problem in today's society. Head injuries occur in about $3.6 \%$ of the population each year (Caveness, 1979), caused by motor vehicle accidents, falls, assaults, industrial accidents, and sports injuries. While mild head injuries are not life-threatening, there is evidence that they contribute to lasting cognitive impairments that can substantially impact work or academic abilities. For instance, deficits in certain types of attention, including information processing speed (Gronwall, 1989, 1991; Gronwall \& Wrightson, 1974, 1975), reaction time (MacFlynn, Montgomery, Fenton, \& Rutherford, 1984; Ponsford \& Kinsella, 1992), divided attention (Gentilini et al., 1985; Stuss et al., 1985; Stuss, Stethem, Hugenholtz, \& Richard, 1989), and working memory (Baddeley, Harris, Sunderland, Watts, \& Wilson, 1987; Newcombe, Rabbitt, \& Briggs, 1994), have been commonly associated with head injuries and may be evident even after apparent recovery (Gentilini et al., 1985; Gronwall \& Wrightson, 1974; Stuss et al., 1985; Stuss et al., 1989). Similarly, long-term memory for verbal information, or declarative memory, has been shown to be impaired following head injuries of varying severity (Levin, Benton, \& Grossman, 1982; Levin \& Goldstein, 1989; Mariadas, Rao, Gangadhar, \& Hegde, 1989; Stuss et al., 1985).

\section{Effects of Glucose on Memory}

Interestingly, head injuries have been likened to the aging process and Alzheimer's disease. Not only do these

This study was supported by a Natural Sciences and Engineering Research Council of Canada grant awarded to R.W.S. Correspondence should be addressed to R. W. Skelton, Department of Psychology, University of Victoria, P. O. Box 3050, Victoria, BC, V8W 3P5, Canada (email: skelton@uvic.ca). conditions have many similar neuropathological characteristics (Cope, 1986), but the psychological characteristics are also comparable. Substantial research evidence indicates that, with age, humans experience a decline in the ability to learn and retain new information (Hock, 1987). Pharmaceutical approaches that augment cognitive deficits resulting from Alzheimer's disease or the aging process may also alleviate these problems in the headinjured. For instance, cognitive enhancing drugs, such as the nootropics, may improve cognition in geriatric and Alzheimer's patients (Hock, 1987), as well as in people who have sustained head injuries (McLean, Cardenas, Burgess, \& Gamzu, 1991). These drugs facilitate learning and retrieval of information (Nicholson, 1990; Wenk, 1989) presumably by increasing the availability of glucose for uptake and utilization in the brain (Wenk, 1989). Recent investigations indicate that glucose alone may enhance cognition in individuals with memory problems. Indeed, researchers have consistently found that glucose administration enhances performance on memory tasks in humans, particularly tasks involving long-term verbal or declarative memory. Ingestion of a glucose-sweetened beverage prior to testing improved retention on the Logical Memory subtest of the Weschler Memory Scale, or a similar type of narrative passage, in elderly individuals (Craft, Murphy, \& Wenstrom, 1994; Gonder-Frederick et al., 1987; Hall, Gonder-Frederick, Chewning, Silveira, \& Gold, 1989; Manning, Hall, \& Gold, 1990; Manning, Parsons, \& Gold, 1992; Parsons \& Gold, 1992) and Alzheimer's patients (Craft, Zallen, \& Baker, 1992; Manning, Ragozzino, \& Gold, 1993). While the mechanism of this performance effect is not known, there is evidence to suggest that glucose ingestion enhances memory storage by preventing future degradation of memory. Manning et al. (1992) demonstrated that, although immediate recall was 
similar in the glucose and saccharin conditions, 24-h recall of that same material was significantly greater under the glucose condition. This effect was apparent regardless of the relative time of administration (preacquisition vs. postacquisition), suggesting that glucose influences storage, rather than acquistion, of information.

The effects of glucose on cognition may depend on individual blood-glucose uptake (i.e., how quickly the bloodglucose level returns to baseline following glucose ingestion). Elderly individuals with memory impairments and Alzheimer's patients tend to have abnormal glucose uptake from the blood and possible impaired brain-glucose utilization (Craft et al., 1992; Gold \& Stone, 1988). Following ingestion of glucose, the large increase in bloodglucose levels might attenuate such an impairment in brain-glucose utilization (Hall et al., 1989) and concomitantly enhance memory. In young, healthy adults, brain glucose is presumably already at an optimal level for memory processes. As a result, blood-glucose levels would not be expected to increase the brain's utilization of glucose and thus enhance memory further. The effects of glucose on memory in head-injured individuals have not been previously reported. We speculated that bloodglucose regulation plays a role.

In the present investigation, we sought to determine whether glucose enhancement of cognition can be extended to include individuals with attention and memory deficits resulting from mild head injury. Our second aim was to investigate the relationship between these cognitive deficits and blood-glucose uptake in these individuals.

\section{Rugby Players, Cognition, and Glucose}

The participants in this experiment were varsity rugby players, both with and without a history of concussions. A substantial percentage of university students involved in contact sports, such as football (Alves, 1991; Alves, Rimel, \& Nelson, 1987; Barth et al., 1989; Reid, Tarkington, Epstein, \& O'Dea, 1971; Willberger, 1993) and rugby (Gronwall, 1992; Ingersoll, 1993; Pettersen, 2000; Sparks, 1985; Wrightson, 1992), have received one or more concussions. In varsity football, the concussion incidence is $42 \%$, with many involving associated attention and memory impairments (Barth et al., 1989). In rugby, the incidence of concussions is believed to be even higher, affecting as many as $50 \%$ of players (Pettersen, 2000 ). Repeated concussions, which may affect as many as $22 \%$ of varsity football players (Barth et al., 1989) and a similarly large percentage of rugby players (Pettersen, 2000; Wrightson, 1992), likely have a cumulative effect on cognitive functioning, leading to a mild head injury (a more chronic condition than concussion). For example, Gronwall and Wrightson (1975) demonstrated that information processing speed was significantly reduced and took longer to recover in individuals who had sustained two concussions relative to individuals who had sustained only one concussion. In addition, it has been reported that repeated concussions sustained in boxing have a cu- mulative effect on overall intellectual performance (Casson et al., 1984; Kaste et al., 1982). These findings imply that repeated concussions (or mild head injuries) may detrimentally affect an athlete's ability to perform well on cognitive tasks and academic studies.

Due to the nature of rugby (i.e., it is a contact sport with the potential for injury, and headgear is not mandatory), we expected that rugby players may be particularly affected by head injuries and associated cognitive problems. The ability of glucose to enhance cognition may be particularly relevant in this population because cognitive deficits are likely quite subtle. The question of this investigation was whether an optimal dose of glucose may be able to enhance cognitive performance in this population to, or near to, the level of controls.

The types of cognitive functions tested in this study were those that were expected to be most susceptible to impairment following mild head injury. These cognitive functions included declarative memory, information processing speed, divided attention, working memory, and passive memory for nonverbal information.

Each participant ingested a beverage sweetened with glucose or saccharin (placebo condition) prior to being tested on the series of neuropsychological tests. The following day, the other sweetener was given, and the participants were tested on alternate forms of the same neuropsychological tests. By having each participant experience both conditions, we could determine the effects of glucose (relative to saccharin) on cognition for each individual. To examine the relationship between head injuries and blood-glucose uptake, blood-glucose levels were monitored for the duration of both testing sessions.

It was expected that participants with a history of concussion would not perform as well as controls on the neuropsychological tasks, at least in the saccharin (placebo) condition. However, we expected that glucose would enhance the performance of previously concussed participants, particularly on a delayed recall declarative memory task. Control participants were not expected to exhibit enhanced performance on these tasks following glucose ingestion.

\section{METHOD}

\section{Participants}

The participants were 24 male undergraduate students from the University of Victoria, who were members of one of the four school rugby teams ( $n=20$ ) or were involved in a similar team sport $(n=$ 4). The participants received either financial compensation $(\$ 10)$ or bonus points toward an introductory psychology course on completion of their participation. The participants ranged in age from 18 to 24 years (control group, $M=19.6$ years; previously concussed group, $M=19.9$ years) and had between 14 and 17 years of education (control group, $M=14.6$ years; previously concussed group, $M=15.3$ years). During a preliminary screening interview, information regarding health was collected from each participant, and it was determined that all participants were nondiabetic and in good health. In addition, the participants were questioned about their injury history, and cognitive functioning was assessed by their per- 
formance on two tests: Information processing speed and passive memory for nonverbal information were assessed with the Symbol Digit Modalities Test (SDMT; Smith, 1968, 1973) with incidental recall, and working memory capacity was assessed with the Listening Span (LS) Test (Salthouse \& Babcock, 1991). The results from the injury history reports and the cognitive tests indicated that 12 participants had no history of head trauma (control group) and that the other 12 participants had received one or more concussions within the past 10 years (previously concussed group). The criterion for inclusion in the previously concussed group included the occurrence of at least one injury involving the head within the last 10 years that resulted in one or more of the following: hospital admission, posttraumatic amnesia, altered level of consciousness, or fracture to the skull. Jennett (1975) reported that using these criteria will include injuries of varying severity yet will exclude many cases in which there is doubt about whether a head injury was sustained at all.

\section{Procedure}

The testing regimen used in this study was adapted from Hall et al. (1989): A repeated measures counterbalanced crossover design was employed in which each participant was tested under two different sweeteners (glucose and saccharin) and two equivalent sets of neuropsychological tests on consecutive days. The participants were individually tested and were blind to sweetener condition.

The participants attended three consecutive morning sessions at the same time each day, between 7:30 a.m. and 11:30 a.m. All participants were instructed to come to the testing room each morning having fasted from midnight the night before. On arrival at the first session, the participants were told that the purpose of the experiment was to evaluate the effects of artificial versus natural sweeteners on attention and memory, and they were briefly introduced to the experimental methods. An informed consent form outlining the purpose and methods was then given to the participants to sign, if they wished to continue participation in the study.

Baseline blood-glucose levels were measured using a Glucoscan One Touch Basic meter (Lifescan, Inc.) at the commencement of all three testing sessions. Blood samples were taken again 15 and $50 \mathrm{~min}$ after beverage ingestion for the first and second testing sessions to assess glucose levels and clearance rates. This procedure, which is virtually painless, involved pricking the participant's finger with a penlet and placing one drop of blood on an enzyme pad, which was then read by a reflectance meter.

After initial measurement of baseline blood-glucose levels, the participants ingested a $16-\mathrm{oz}$ lemon-lime-flavored beverage sweetened with either saccharin (47.4 mg) or glucose $(50 \mathrm{~g}$, with $23.7 \mathrm{mg}$ saccharin added to equate for taste). Beginning 3 min after drinking the beverage, the participants were tested on the following series of neuropsychological tests.

Logical memory scale (LMS). This subtest of the Wechsler Memory Scale-Revised (Russell, 1975) was used to assess declarative memory. Prior to listening to a narrative passage containing 25 units of information (phrases), the participants were notified that they would be asked to recall the passage both immediately after presentation and $24 \mathrm{~h}$ later.

The following day, 24-h recall was tested without repetition of the narrative passage. Recall was measured as the total number of correctly recalled units, each worth one point.

Paired associates (PA). This test was used to assess noncontextual declarative memory. Two equivalent sets of eight word pairs each were constructed from an original set of 30 word pairs (Jones, 1974) using associative difficulty ratings provided by 25 university students. Prior to listening to a list of eight word pairs (five of which were concrete and three of which were abstract), the participants were notified that they would be asked to recall the word pairs immediately after presentation and again $24 \mathrm{~h}$ later. After the list was read out, the first word in each pair was presented and the participants were asked to recall its associate. The following day, $24-\mathrm{h}$ re- call was tested without repetition of the list. One point was given for each correct answer.

Auditory consonant trigrams (CCC). This task (Brown, 1958; Peterson \& Peterson, 1959), which tests recall of auditory information under conditions of interference, was used to assess divided attention. On hearing three consonants followed by a number, the participants were to immediately begin counting backward from that number by threes until instructed to stop (i.e., after a delay of 0,9 , 18 , or $36 \mathrm{sec}$ ). At this time, the participants were asked to recall the three consonants. Five trials at each of four time delays were administered. One point was given for each correctly recalled letter.

Symbol Digit Modalities Test (SDMT). This test, developed by Smith $(1968,1973)$, was used to assess information processing speed. The participants were given a "key" with nine symbols, each corresponding to a number from 1 to 9 . During a $90-\mathrm{sec}$ trial, the participant was to write in the correct number that corresponded to each given symbol, working quickly and accurately to correctly complete as many of the 110 items as possible. One point was given for each correct answer.

SDMT Incidental Recall (SDMT-D). This test was used to assess passive memory for nonverbal material. Following a 5-min filled delay, in which the LMS was administered, the participants were asked to complete 15 items from memory.

ANAM Battery. The following four tests, which are part of the Automated Neuropsychological Assessment Metrics (ANAM) battery (Kane \& Kay, 1992; Reeves, Thorne, Winter, \& Hegge, 1989) were administered via a 386 DX IBM-compatible computer with a three-button mouse. Accuracy, response time, and efficiency (a composite measure of accuracy and speed) were recorded.

Simple Reaction Time (SRT). This test measures the speed of reaction to a single stimulus and thereby assesses a low level of information processing. The participants were to press the left button on the computer mouse as quickly as possible following the presentation of an asterisk on the computer screen. The test, which included a list of instructions, five practice trials, and 25 test trials, took about $1 \mathrm{~min}$ to complete.

Two-Choice Reaction Time (2-CH). This test measures speed of two different reactions to two different stimuli, thereby assessing a slightly higher level of information processing than simple reaction time. The computer randomly displayed one of two different symbols (an asterisk or a cross). The participants were to respond by quickly pressing the left button if the asterisk was shown and the right button if the cross was displayed. Instructions and five practice trials preceded the 45 test trials, with the entire test taking about 3 min to complete.

Running Memory Continuous Performance Test (RM-CPT). This test was used to assess information processing speed. The computer displayed a random series of letters (one at a time) to which the participant was to respond by pressing the left button if the letter being shown matched the one that came immediately before it or the right button if the letter being shown was different from the previous letter. This test included a list of instructions, 10 practice trials, and 160 test trials, which took a total of about $5 \mathrm{~min}$ to complete.

Tower of Hanoi Test. This test was used to assess working memory. The computer displayed three posts, each of which had some blocks of varying sizes stacked on them. The object of the task was to place all of the blocks on the middle post, with the largest block on the bottom and successively smaller blocks stacked so that the smallest block was at the top of the stack. Blocks could be moved, one at a time, to another post (by pressing two number keys on the keyboard, corresponding to the number of the source and target posts), with the condition that larger blocks could not be placed on top of smaller blocks. The participants were given a list of instructions, two practice tests, and six test trials, each taking a maximum of $2 \mathrm{~min}$

The first session lasted about $1 \mathrm{~h}$. It began with determining baseline blood-glucose levels and then beverage administration. 
The neuropsychological tests were then administered in the following order: CCC, PA, SRT, 2-CH, RM-CPT, SDMT, LMS, SDMT-D, Tower of Hanoi.

Long-term (24-h) retention of items from the LMS and the PA test was assessed the following morning, at the start of the second session. The second testing day was identical to the first, except the other beverage condition (glucose or saccharin) was tested, and equivalent versions of the same neuropsychological tests (except for the $\mathrm{CCC}$, in which the same version was used on both testing days) were employed.

On the third testing day, long-term retention of items from the previous day's versions of LMS and PA tasks was tested, and the participants were then debriefed and thanked for their participation in the study. Debriefing consisted of providing the participants with additional information about the hypotheses and requesting that they not disclose this or the contents of the tests to other potential participants. The participants were also asked to guess the identity of each drink (artificially vs. naturally sweetened) and to comment on their perception of the cognitive and performance effects of each drink and also the presence of extenuating circumstances that might have affected their performance (e.g., amount of sleep the previous night).

\section{Statistics}

To assess the presence of deficits from concussions, $t$ tests were used to compare the performance of previously concussed participants with that of controls on the screening interview tests and the tests given in the saccharin condition. Given the clear unidirectional hypothesis that concussed participants would do worse, one-tailed $t$ tests were used to compare differences in blood-glucose levels between the two groups. Also, paired $t$ tests were used to examine changes in blood-glucose levels over time. A repeated measures analysis of variance (ANOVA) was conducted to examine the main effects of glucose versus saccharin on test performance in the two groups. Pearson correlations were used to investigate the relationship between the number of concussions and performance on the LMS-D in the saccharin condition and between blood-glucose recovery and difference scores (glucose-saccharin) for 24 -h recall of the LMS and LMS-SAV. Finally, a chi-square analysis was used to determine whether the participants were aware of the correct identity of each drink. Due to the number of statistical tests used in the present investigation, we recognize that there is an increased risk of committing a Type I error. Therefore, higher $p$ values, particularly those with values of .01 or higher, should be regarded cautiously.

\section{RESULTS}

The screening interview revealed that the number of concussions per participant ranged from 0 to 14 . Those with at least one concussion, as a group, had a mean of $4.33(S D=3.55)$ and a modal number of 2 concussions. Interestingly, 11 of the 12 previously concussed participants had experienced at least two concussions. Duration of loss of consciousness and posttraumatic amnesia was difficult to ascertain accurately; however, duration of loss of consciousness seemed to range from no loss of consciousness to $1 \mathrm{~min}$, and that of posttraumatic amnesia ranged from $1 \mathrm{~min}$ to a few hours. Comparison of the performance of previously concussed participants with that of controls on all tests for the screening interview and saccharin condition revealed selective and subtle deficits. The previously concussed participants scored slightly lower than did the controls on four measures: SDMT-D, all measures of the LS (LS-SPAN, LS-TRIAL, LS-ITEM; see Figure 1), the CCC, and the LMS-D (see Figure 2) ( $t$ tests, $p<.05$, one-tailed). However, previously concussed participants did not differ from controls with respect to performance on the SDMT, PA-I, PA-D, LMS-I, or any test from the ANAM battery. There appeared to be a cumulative effect of concussions on memory: The number of concussions per participant was significantly cor-

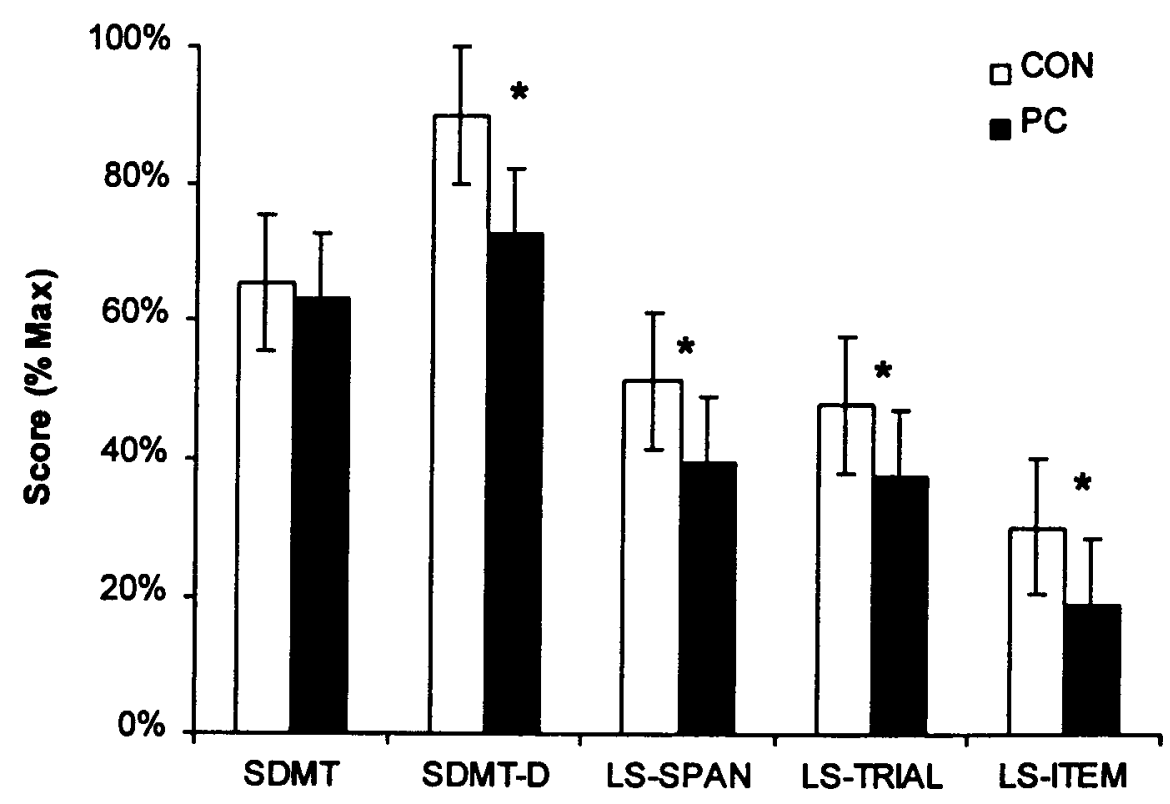

Figure 1. The differences between the previously concussed (PC) and control (CON) groups for percent correct on screening interview tests. SDMT, Symbol Digit Modalities Test; SDMTD, Symbol Digit Modalities Test with delayed incidental recall; LS, Listening Span. ${ }^{*} p<.05$. 


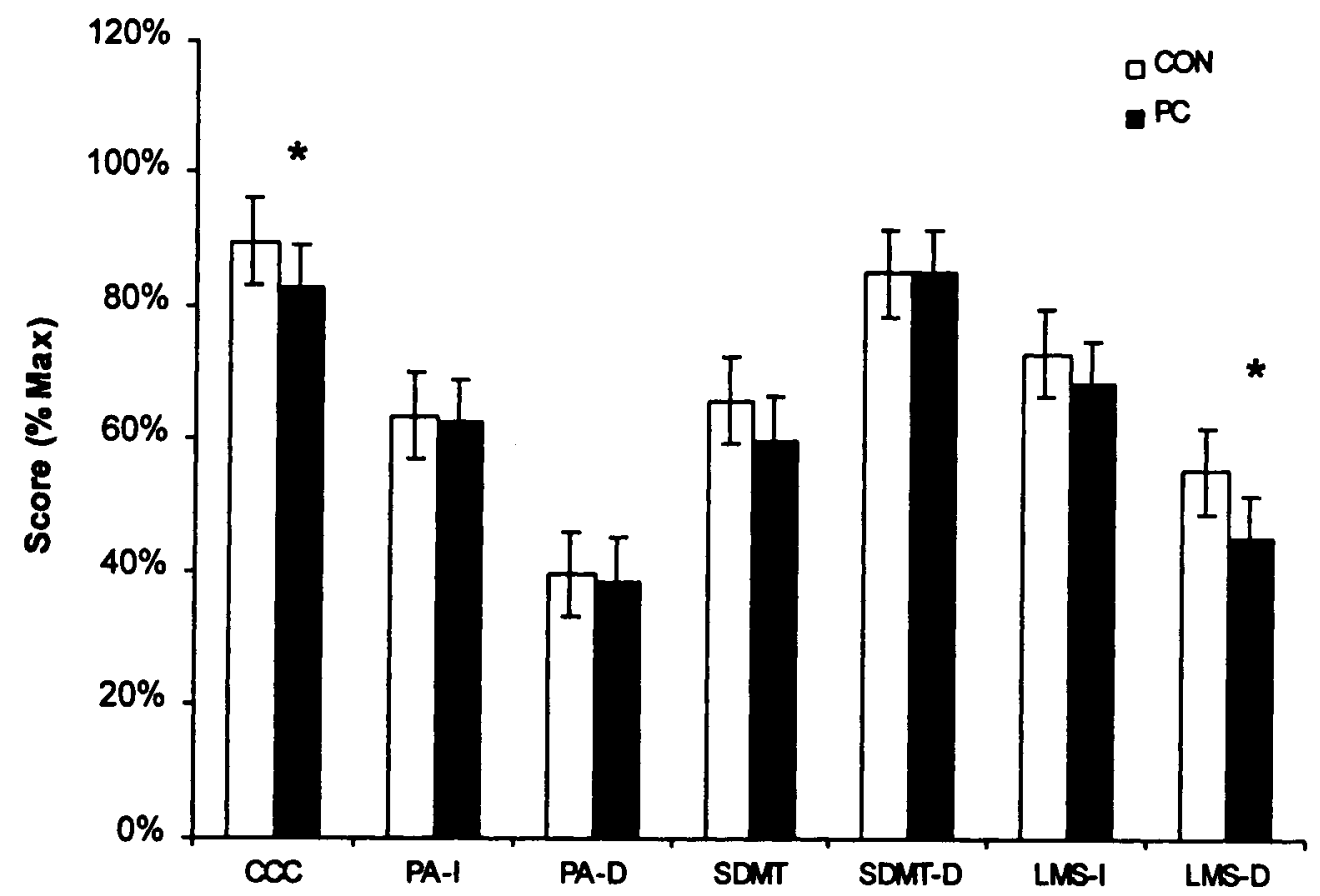

Figure 2. The differences between the previously concussed (PC) and control (CON) groups for percent correct on tests given on the saccharin (placebo) day. CCC, consonant trigrams test; PA-I, Paired Associates task with immediate recall; PA-D, Paired Associates task with delayed recall; SDMT, Symbol Digit Modalities Test; SDMT-D, Symbol-Digit Modalities Test with delayed incidental recall; LMS-I, Logical Memory Scale with immediate recall; LMS-D, Logical Memory with delayed recall. $\quad$ * $p<.05$.

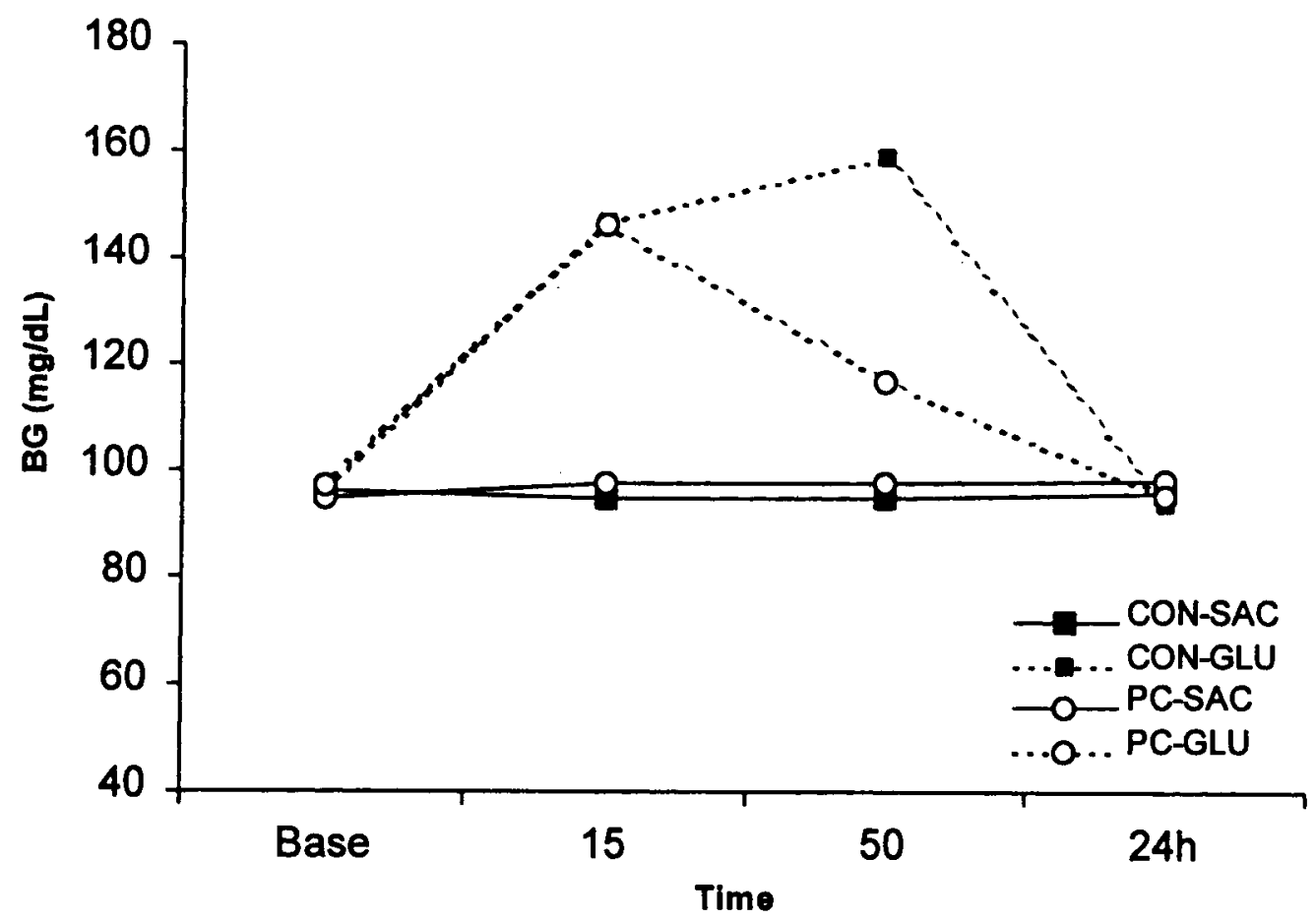

Figure 3. Group mean blood-glucose levels for each monitoring point throughout the testing sessions. CON, control; PC, previously concussed; SAC, saccharin; GLU, glucose; BG, blood-glucose. 
related with poorer performance on the LMS-D in the saccharin (placebo) condition (Pearson $r=-.615, p<.033$ ).

Blood-glucose readings from the previously concussed and control groups for the glucose and saccharin conditions are compared in Figure 3. There were no significant differences between the control and previously concussed groups on baseline blood-glucose levels for the saccharin or the glucose day. While the saccharin beverage did not change blood-glucose levels from baseline in either of the two groups (all $p \mathrm{~s}>.05$ ), ingestion of $50 \mathrm{~g}$ glucose significantly elevated blood-glucose levels in both groups at 15 min postingestion (paired $t$ tests between baseline and 15 -min levels, $p s<.001$ ). Peak bloodglucose change (maximum of $15 \mathrm{~min}$ or $50 \mathrm{~min}$ ) was not significantly different between the two groups $[t(22)=$ $1.81, p<.08$, n.s.]. However, at $50 \mathrm{~min}$, the blood-glucose level was still high in the control group, but had dropped significantly in the previously concussed group [paired $t$ test between 15- and 50-min levels within the previously concussed group, $t(11)=3.60, p<.004]$ and was significantly lower than the level of the controls $[t(11)=$ $2.76, p<.01]$.

As shown by performance on the LMS measures, glucose significantly influenced long-term declarative memory, although in different ways in the previously concussed and control groups: Glucose enhanced long-term declarative memory in the previously concussed group, whereas it impaired long-term declarative memory in the control group. A comparison of the two groups on the difference in sweetener condition (saccharin, glucose) for performance on the LMS-D revealed a significant interaction of sweetener and concussion $[F(1,22)=11.46$, $p<.003]$. The previously concussed group scored significantly higher on the glucose day relative to on the saccharin day [paired $t(11)=-3.39, p<.007$ ], whereas the controls scored significantly lower [paired $t(11)=-2.29$, $p<.04]$. As Figure 4 shows, glucose enhanced long-term declarative memory in the previously concussed group almost to the level of the controls in the saccharin condition. To examine whether glucose's effect on memory

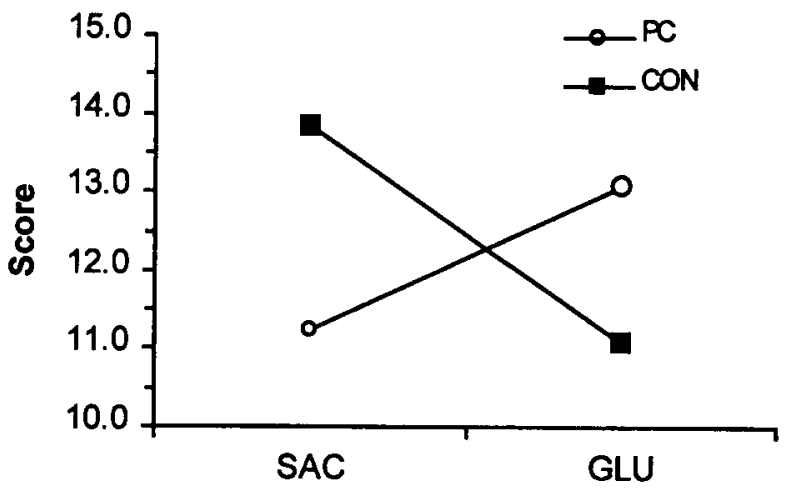

Figure 4. Performance on the Logical Memory Scale-Delayed (LMS-D) of previously concussed (PC) and control (CON) participants on glucose (GLU) and saccharin (SAC) days.

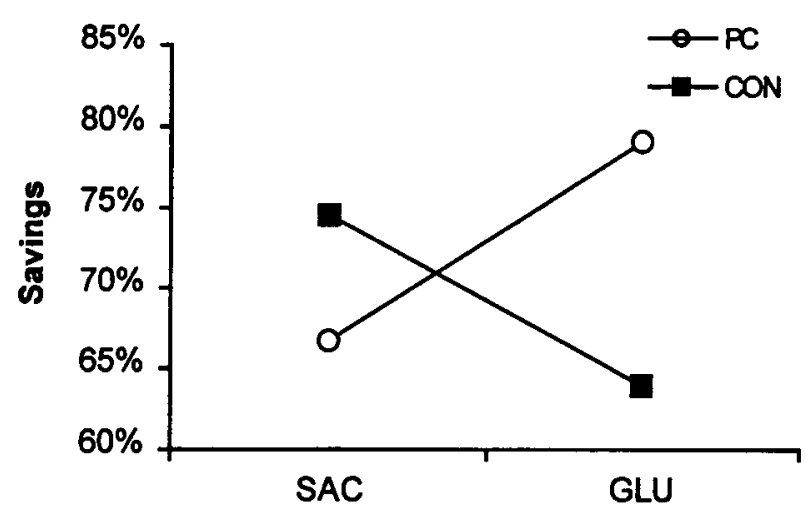

Figure 5. Savings on the Logical Memory Scale. Performance on the delayed test (LMS-D) as a percentage of performance on the immediate test (LMS-I) for previously concussed (PC) and control (CON) participants on glucose (GLU) and saccharin (SAC) days.

was due to a change in retention, proportional savings scores were calculated to compare test performance immediately after learning to test performance $24 \mathrm{~h}$ later [1 - (LMS-I - LMS-D)/LMS-I]. The higher the proportional savings score, the greater the amount of information retained. A repeated measures ANOVA comparing glucose and saccharin condition scores of the two groups on the LMS-SAV revealed a significant interaction $[F(1,22)=7.84, p<.01]$ (see Figure 5). The controls retained significantly less information from the glucose day than from the saccharin day [paired $t$ test on LMS-SAV within control group, $t(11)=2.15, p<.05]$. In contrast, the previously concussed participants tended to retain more information from the glucose day than from the saccharin day [paired one-tailed $t$ test within head-injured group, $t(11)=3.04, p<.04]$. In fact, on the day after glucose, the previously concussed group retained as much as, if not more than, the control group [ $t$ test on differences between control and previously concussed groups on LMS-SAV, glucose day, $t(22)=1.96, p<.06$, n.s.].

There was no evidence that glucose affected information processing speed (four measures), divided attention, passive memory for nonverbal information, working memory, short-term and long-term noncontextual declarative memory, and short-term contextual declarative memory, in that there were no significant differences between performances in the glucose and saccharin conditions for SRT, 2-CH, SDMT, RM-CPT, CCC, SDMT-D, Tower of Hanoi, PA (immediate and 24-h recall), and the immediate recall of the LMS, respectively (all $p s>.05$ ). However, subtle glucose effects on divided attention and information processing speed may have been masked by unexpected but significant practice effects on the CCC [paired $t$ test, $t(23)=-4.85, p<.001$ ] and unexpected differences between the two versions of the SDMT [paired $t$ test, $t(23)=-4.67, p<.001]$, respectively.

Blood-glucose recovery (i.e., the change in bloodglucose readings from $15 \mathrm{~min}$ to $50 \mathrm{~min}$ postingestion of 
the glucose beverage), which tended to be quicker in the previously concussed group than in the control group, was significantly correlated with glucose enhancement of memory. In particular, the participants with quicker bloodglucose recovery remembered more on the 24 -h recall of the LMS-D when given glucose rather than saccharin: Blood-glucose recovery (15-50 min) was significantly correlated with difference scores (glucose-saccharin) for $24-\mathrm{h}$ recall of the LMS $(r=.55, p<.006)$ and with difference scores on the LMS-SAV $(r=-.56, p<.005)$. This suggests that glucose uptake from the blood was more important than blood-glucose levels per se.

The debriefing session revealed that prior knowledge of the research hypotheses would not have influenced performance, since the participants could not correctly identify which drink was sweetened with glucose. A chisquare analysis revealed that the number of correct guesses was not significantly different from chance $\left[\chi^{2}(1, N=\right.$ 24) $=0.17, p=.88]$.

\section{DISCUSSION}

In general, the present investigation confirmed that subtle residual deficits in long-term declarative memory and other types of cognition result from concussions. It also showed that glucose selectively alleviated deficits in long-term declarative memory in previously concussed individuals. The effects of glucose on memory were positively related to ability to clear glucose from the blood.

\section{Head Injuries and Cognitive Impairments}

In the present study, previously concussed participants appeared to have subtle deficits in long-term declarative memory, scoring lower than controls on the LMS-D (in the saccharin condition). This effect extends previous findings that head injuries impair performance on the LMSD (Mateer, 1992; Stuss et al., 1985) and on other measures of long-term declarative memory (Levin et al., 1982; Lezak, 1979; Mariadas et al., 1989). Our results support the assertion that memory is one of the cognitive functions most susceptible to impairment by closed head injury (Capruso \& Levin, 1992; Levin, Goldstein, High, \& Eisenberg, 1988). As well, divided attention, passive memory for nonverbal material, and working memory appeared to be impaired following concussions: Previously concussed participants scored slightly lower than controls on the CCC (in the saccharin condition), the SDMT-D, and the Listening Span Test (both from the screening interview). Although there is no strong evidence in the literature to support our latter two findings, our finding that deficits in divided attention may result from head injuries is in accordance with the findings of Stuss and colleagues (Stuss et al., 1985; Stuss et al., 1989).

Concussions appeared to have a cumulative effect on long-term declarative memory. There was a negative correlation between the number of concussions and the scores on the LMS-D in the saccharin (placebo) condition, such that participants with more concussions recalled fewer details of a narrative passage. Although we are not aware of previous reports of this effect in sports other than boxing (Casson et al., 1984; Kaste et al., 1982), our finding extends the finding of a cumulative effect of concussions on information processing speed (Gronwall \& Wrightson, 1975) and supports the often-believed notion that repeated concussions can lead to loss of memory function.

\section{Effects of Glucose on Memory}

Glucose was found to influence long-term declarative memory but had different effects on the two groups: It enhanced long-term declarative memory in previously concussed participants, whereas it impaired long-term declarative memory in controls. The previously concussed group obtained higher LMS-D scores after ingesting glucose than after ingesting saccharin. In fact, previously concussed participants given glucose, who were slightly impaired on the LMS-D in the saccharin condition, actually retained as much information as (if not more than) the control group in the glucose condition. This glucoseenhancement of memory extends previous reports of glucose selectively enhancing declarative memory in individuals with memory impairments, such as Alzheimer's patients and elderly individuals (Craft et al., 1992; Gonder-Frederick et al., 1987; Hall et al., 1989; Manning et al., 1990; Manning, Parsons, Cotter, \& Gold, 1997; Manning et al., 1992; Manning et al., 1993; Parsons \& Gold, 1992).

Previous studies have found negligible memoryenhancing effects of glucose in young individuals (Azari, 1991; Hall et al., 1989; Manning et al., 1997) or even slight impairments (Craft et al., 1994; Craft et al., 1992). The latter authors suggested that the rate of glucose uptake from the blood may determine whether glucose will help or hinder memory. Conceivably, fast glucose uptake may hinder memory in controls by overstimulating a physiological memory consolidation mechanism beyond optimal functioning, or, alternatively, it could simply enhance consolidation so much that proactive interference with subsequent recall is detrimental to performance.

In the present investigation, glucose uptake from the blood was correlated with glucose enhancement of memory and differed between the two groups, with previously concussed participants showing faster glucose uptake. Previous studies concerning the relationship between blood-glucose uptake and memory enhancement were inconclusive. While it has been suggested that no relationship exists between glucoregulatory control and memory performance in young adults (Hall et al., 1989), others have demonstrated that blood-glucose regulation is correlated with the effects of glucose on memory, though the direction of this correlation varied between investigations. In one study, subjects who had fast glucose uptake from the blood showed enhanced memory following ingestion of glucose, whereas subjects with slower glucose uptake showed memory deterioration (Craft et al., 1992). In another study, recall on a declarative memory measure was enhanced in older males with fast glucose 
uptake and in young males with slow uptake, whereas it was impaired in young males with fast uptake (Craft et al., 1994). Differences in findings may well be due to differences in peak blood-glucose levels. In our study, the blood-glucose levels of participants with fast uptake reached about the same peak levels (about $140 \mathrm{mg} / \mathrm{dL}$ ) as did the young subjects with slow uptake in the investigation by Craft et al. (1994), and both of these groups showed enhanced memory. Thus, glucose enhancement of memory appears to be associated not only with the rate of uptake but also with optimal levels of blood glucose. Taken together, these studies suggest that glucose enhances performance on long-term declarative memory tasks in an inverted-U response manner (Parsons \& Gold, 1992) and suggest that optimal doses will depend on an individual's glucoregulatory mechanisms.

In our study, the memory-enhancing effect of glucose outlasted its presence in the blood. However, glucose could still be causal, since the time course of glucose's actions remains unknown. If glucose acts indirectly (e.g., by facilitating synthesis of acetylcholine), its effects could persist well after it has been taken up from the blood. Indeed, rapid uptake might improve its actions.

The glucose effect seemed to be selective to memory processes themselves, since glucose did not appear to affect other aspects of cognitive functioning, including information processing speed, divided attention, passive memory for nonverbal information, working memory, noncontextual declarative memory (immediate or delayed), or immediate contextual declarative memory, which could have indirectly improved memory consolidation.

It is not clear why the previously concussed participants had faster glucose uptake than controls. Although all participants in our study were athletes, individuals with a history of concussions may have been more committed to rugby (i.e., having played longer period of time, or playing and training more intensely) and, accordingly, were more physically fit than controls. Level of physical fitness is highly correlated with insulin-stimulated glucose utilization (Rosenthal, Hashell, Solomon, Widstrom, \& Reaven, 1983). It does not, however, elucidate whether long-term declarative memory would be enhanced by glucose only in individuals with faster glucose uptake, regardless of head injury status.

One possible explanation for the relationship between concussion and glucose uptake is very speculative. Head injury is known to increase mental effort (Gronwall, 1989), and this might lead to a chronically greater demand for glucose by the brain. This, in turn, might lead to an up-regulation of glucose transport mechanisms to compensate. In this way, concussed participants in our study might have been better able to use the high blood glucose for mental processing. The possibility that glucose regulation plays a role in head injury deficits would seem to warrant further investigation.

Our results suggest that consolidation of declarativetype memories may depend on a glucose-mediated neurochemical process. Although the mechanisms underly- ing glucose's effects on memory are not entirely known, it has been proposed that circulating glucose levels might influence cerebral neurotransmitters, such as acetylcholine (Gold, 1991; Kopf \& Baratti, 1994; Messier, Durkin, Mrabet, \& Destrade, 1990, 1991). Recent evidence suggests that glucose facilitates acetylcholine synthesis particularly in the hippocampus (Messier et al., 1990, 1991), a brain structure strongly implicated in the storage and retrieval of new memories (Squire \& Zola-Morgan, 1991). Thus, the effects of glucose may be primarily limited to hippocampal-mediated cognitive functions. An important implication of our findings is that other methods of pharmaceutical therapy that alleviate long-term declarative memory problems in Alzheimer's patients and the elderly may have a similar effect in mildly headinjured individuals.

\section{REFERENCES}

ALvEs, W. M. (1991). Football-induced mild head injury. In J. S. Torg (Ed.), Athletic injuries to the head, neck and face (2nd ed., pp. 283304). St. Louis: Mosby.

Alves, W. M., Rimel, R. W., \& Nelson, W. E. (1987). University of Virginia prospective study of football-induced minor head injury: Status report. Head \& Neck Injuries, 6, 211-218.

AZARI, N. P. (1991). Effects of glucose on memory processes in young adults. Psychopharmacology, 105, 521-524.

Baddeley, A., Harris, J., Sunderland, A., Watts, K. P., \& Wilson, B. A. (1987). Closed head injury and memory. In H. S. Levin, J. Grafman, \& H. M. Eisenberg (Eds.), Neurobehavioral recovery from head injury (pp. 295-317). New York: Oxford University Press.

Barth, J. T., Alves, W. M., Ryan, T. V., Macciocchi, S. N., Rimel, R. W., JANE, J. A., \& NelSON, W. E. (1989). Mild head injury in sports: Neuropsychological sequelae and recovery of function. In H. S. Levin, H. M. Eisenberg, \& A. L. Benton (Eds.), Mild head injury (pp. 255-275). New York: Oxford University Press.

BRown, J. (1958). Some tests of the decay theory of immediate memory. Quarterly Journal of Experimental Psychology, 10, 12-21.

Capruso, D. X., \& Levin, H. S. (1992). Cognitive impairment following closed head injury. Neurology of Trauma, 10, 879-893.

Casson, I. R., Siegel, O., Sham, R., Campbell, E., Tarlau, M., \& DiDomenico, A. (1984). Brain damage in modern boxers. Journal of the American Medical Association, 251, 2663-2667.

CAVENESS, W. (1979). Incidence of craniocerebral trauma in the United States in 1976 with trend from 1970 to 1975. Advances in Neurology, 22, 1-3.

COPE, D. N. (1986). The pharmacology of attention and memory. Journal of Head Trauma Rehabilitation, 1, 34-42.

Craft, S., Murphy, C., \& Wemstrom, J. (1994). Glucose effects on complex memory and nonmemory tasks: The influence of age, sex, and glucoregulatory response. Psychobiology, 22, 95-105.

Craft, S., Zallen, G., \& Baker, L. D. (1992). Glucose and memory in mild senile dementia of the Alzheimer type. Journal of Clinical \& Experimental Neuropsychology, 14, 253-267.

Gentilini, M., Nichelli, P., Schoenhuber, R., Bartolotti, P., Tonelli, L., Falasca, A., \& Merli, G. A. (1985). Neuropsychological evaluation of mild head injury. Journal of Neurology, Neurosurgery, \& Psychiatry, 48, 137-140.

GoLD, P. E. (1991). An integrated memory regulation system: From blood to brain. In R. Frederickson, J. McGaugh, \& D. Felton (Eds.), Peripheral signaling of the brain (pp. 391-419). Toronto: Hogrefe \& Huber.

Gold, P. E., \& Stone, W. S. (1988). Neuroendocrine effects on memory in aged rodents and humans. Neurobiology of Aging, 9, 709-717.

Gonder-Frederick, L., Hall, J. L., Vogt, J., Cox, D. J., Green, J., \& GoLD, P. E. (1987). Memory enhancement in elderly humans: Effects of glucose ingestion. Physiology \& Behaviour, 41, 503-504. 
Gronwall, D. (1989). Cumulative and persisting effects of concussion on attention and cognition. In H. S. Levin, H. M. Eisenberg, \& A. L. Benton (Eds.), Mild head injury (pp. 153-162). New York: Oxford University Press.

GronWALL, D. (1991). Minor head injury. Neuropsychology, 5, 253-265.

Gronwall, D. (1992). Head injury in rubgy. New Zealand Journal of Sports Medicine, 20, 19-21.

Gronwall, D., \& Wrightson, P. (1974). Delayed recovery of intellectual function after minor head injury. The Lancet, 2, 605-609.

Gronwall, D., \& Wrightson, P. (1975). Cumulative effect of concussion. The Lancet, 2, 995-997.

Hall, J. L., Gonder-Frederick, L. A., Chewning, W. W., SilVEIRA, J., \& Gold, P. E. (1989). Glucose enhancement of performance on memory tests in young and aged humans. Neuropsychologia, 27, 1129-1138.

HoCk, F. J. (1987). Drug influences on learning and memory in aged animals and humans. Neuropsychobiology, 17, 145-160.

INGERSOLL, C. D. (1993). Long-term effects of head injuries in sports. Sports Medicine, 16, 342-354.

JENNETT, B. (1975). Epilepsy after non-missile head injuries (2nd ed.). London: William Heinemann.

JONES, M. K. (1974). Imagery as a mnemonic aid after left temporal lobectomy: Contrast between material-specific and generalized memory disorders. Neuropsychologia, 12, 21-30.

KANE, R. L., \& KAY, G. G. (1992). Computerized assessment in neuropsychology. Neuropsychological Review, 3, 45-55.

Kaste, M., Vil.kki, J., Sainio, K., KuUrne, T., Katevuo, K., \& MeURALA, H. (1982). Is chronic brain damage in boxing a hazard of the past? The Lancet, 2, 1186-1188.

KOPF, S. R., \& BARATTI, C. M. (1994). Memory-improving actions of glucose: Involvement of a central cholinergic muscarinic mechanism. Behavioral \& Neural Biology, 62, 237-243.

Levin, H. S., Benton, A. L., \& Grossman, R. G. (1982). Neurobehavioral consequences of closed head injury. New York: Oxford University Press.

Levin, H. S., \& Goldstein, F. C. (1989). Neurobehavioral aspects of traumatic brain injury. In P. Bach-y-Rita (Ed.), Comprehensive neurologic rehabilitation: Vol. 2. Traumatic brain injury (pp. 53-72). New York: Demos.

Levin, H. S., Goldstein, F. C., High, W. M., JR., \& Eisenberg, H. M. (1988). Disproportionately severe memory deficit in relation to normal intellectual functioning after closed head injury. Journal of Neurology, Neurosurgery, \& Psychiatry, 51, 1294-1301.

LEZAK, M. D. (1979). Recovery of memory and learning functions following traumatic brain injury. Cortex, 15, 63-72.

MacFlynn, G., Montgomery, E. A., Fenton, G. W., \& RutherFORD, W. (1984). Measurement of reaction time following minor head injury. Journal of Neurology, Neurosurgery, \& Psychiatry, 47, 1326-1331.

Manning, C. A., Hall, J. L., \& Gold, P. E. (1990). Glucose effects on memory and other neuropsychological tests in elderly humans. Psychological Science, 1, 307-311.

Manning, C. A., Parsons, M. W., Cotter, E. M., \& Gold, P. E. (1997). Glucose effects on declarative and nondeclarative memory in healthy elderly and young adults. Psychobiology, 25, 103-108.

Manning, C. A., Parsons, M. W., \& Gold, P. E. (1992). Anterograde and retrograde enhancement of 24-h memory by glucose in elderly humans. Behavioral \& Neural Biology, 58, 125-130.

Manning, C. A., Ragozzino, M. E., \& Gold, P. E. (1993). Glucose enhancement of memory in patients with probable senile dementia of the Alzheimer's type. Neurobiology of Aging, 14, 523-528.

Mariadas, A. C., Rao, S. L., Gangadhar, B. N., \& Hegde, A. S. (1989). Neuropsychological functioning in postconcussion syndrome. NIMHANS Journal, 7, 37-41.

Mateer, C. A. (1992). Systems of care for post-concussive syndrome. In L. J. Horn \& N. D. Zasler (Eds.), Rehabilitation of post-concussive disorders (Vol. 6, pp. 143-160). Philadelphia: Hanley \& Belfus.

Mclean, A., Cardenas, D. D., Burgess, D., \& Gamzu, E. (1991). Placebo-controlled study of pramiracetam in young males with memory and cognitive problems resulting from head injury and anoxia. Brain Injury, 5, 375-380.
Messier, C., Durkin, T., Mrabet, O., \& Destrade, C. (1990). Memoryimproving action of glucose: Indirect evidence for facilitation of hippocampal acetylcholine synthesis. Behavioural Brain Research, 39, 135-143.

Messier, C., Durkin, T., Mrabet, O., \& Destrade, C. (1991). Contribution of hippocampal acetylcholine synthesis to the memoryimproving action of glucose. In R. Frederickson, J. McGaugh, \& D. Felten (Eds.), Peripheral signaling of the brain (pp. 473-477). Toronto: Hogrefe \& Huber.

Newcombe, F., RABBiTT, P., \& Briggs, M. (1994). Minor head injury: Pathophysiological or iatrogenic sequelae? Journal of Neurology. Neurosurgery, \& Psychiatry, 57, 709-716.

Nicholson, C. D. (1990). Pharmacology of nootropics and metabolically active compounds in relation to their use in dementia. Psychopharmacology, 101, 147-159.

Parsons, M. W., \& Gold, P. E. (1992). Glucose enhancement of memory in elderly humans: An inverted-U dose-response curve. Neurobiology of Aging, 13, 401-404.

Peterson, L. R., \& Peterson, M. J. (1959). Short-term retention of individual verbal items. Journal of Experimental Psychology, 58, 193-198.

PetTersen, J. A. (2000). Prevention of concussions in rugby players: Should protective headgear be worn? Manuscript in preparation.

Ponsford, J., \& Kinsella, G. (1992). Attentional deficits following closed-head injury. Journal of Clinical \& Experimental Neuropsychology, 14, 822-838.

Reeves, D., Thorne, R., Winter, S., \& Hegge, F. (1989). The United Tri-Service Cognitive Performance Assessment Battery (UTC-PAB) (U.S. Naval Aerospace Medical Research Laboratory and Walter Reed Army Institute of Research, Rep. No. 89-1). Washington, DC: U.S. Government Printing Office.

Reid, S. E., TARkington, J. A., Epstein, H. M., \& O'DeA, T. J. (1971). Brain tolerance to impact in football. Surgery, Gynecology \& Obstetrics, 133, 929-936.

Rosenthal, M., Hashell, W. L., Solomon, R., Widstrom, A., \& REAVEN, G. M. (1983). Demonstration of a relationship between level of physical training and insulin-stimulated glucose utilization in normal humans. Diabetes, 32, 408-411.

RUSSELL, E. W. (1975). WMS-R: A multiple scoring method for the assessment of complex memory functions. Journal of Consulting \& Clinical Psychology, 43, 800-809.

Salthouse, T. A., \& Babcock, R. L. (1991). Decomposing adult age differences in working memory. Developmental Psychology, 27, 763. 776.

Smith, A. (1968). The Symbol Digit Modalities Test: A neuropsychologic test for economic screening of learning and other cerebral disorders. Learning Disorders, 3, 83-91.

SмIтн, A. (1973). Symbol Digit Modalities Test manual. Los Angeles: Western Psychological Services.

SParKS, J. P. (1985). Rugby football injuries 1980-1983. British Journal of Sports Medicine, 19, 71-75.

SQuire, L. R., \& Zol.A-Morgan, S. (1991). The medial temporal lobe memory system. Science, 253, 1380-1386.

Stuss, D. T., Ely, P., Hugenholtz, H., Richard, M. T., LaRochelle, S., PoIRIER, C .A., \& BELL, I. (1985). Subtle neuropsychological deficits in patients with good recovery after closed head injury. Neurosurgery, 17, 41-47.

Stuss, D. T., Stethem, L. L., Hugenholtz, H., \& Richard, M. T. (1989). Traumatic brain injury: A comparison of three clinical tests, and analysis of recovery. The Clinical Neurologist, 3, 145-156.

WENK, G. L. (1989). An hypothesis on the role of glucose in the mechanism of action of cognitive enhancers. Psychopharmacology, 99, 431-438.

WILLBERGER, J. E. (1993). Minor head injuries in American football. Sports Medicine, 15, 338-343.

Wrightson, P. (1992). Head injury in rugby. New Zealand Journal of Sports Medicine, 20, 18-19.

(Manuscript received September 2, 1998; revision accepted for publication September 3, 1999.) 